\title{
Alkaline Earth Metal Modified H-Mordenites. Their Catalytic Properties in the Isopropylation of Biphenyl
}

Yoshihiro Sugi, ${ }^{1,2, *}$, Hiroshi Tamada, ${ }^{1}$ Akiko Kuriki, ${ }^{1}$ Kenichi Komura, ${ }^{1}$ Yoshihiro Kubota, ${ }^{1,3}$ Stalin Joseph, ${ }^{2}$ Anand Chokkalingam, ${ }^{2}$ Mohamed El. Newehy, ${ }^{4}$ Salem S. Al-Deyab, ${ }^{4}$ Hoi-Gu Jang, ${ }^{5}$ Jong Ho Kim, ${ }^{5}$ Gon Seo, ${ }^{5}$ and Ajayan Vinu ${ }^{2,6, *}$

1. Department of Materials Science and Technology, Faculty of Engineering, Gifu University, Gifu 501-1193, Japan.

2. Australian Institute of Bioengineering and Nanotechnology, The University of Queensland, Brisbane 4072 QLD, Australia.

3. Department of Materials Science and Engineering, Graduate School of Engineering, Yokohama National University, Yokohama 240-8501, Japan.

4. Petrochemical Research Chair, College of Science, Department of Chemistry, King Saud University, Riyadh 11451, Saudi Arabia.

5. School of Applied Chemical Engineering, Chonnam National University, Gwangju, 500-757, Korea.

6. Future Industries Institute, The University of South Australia, Mawson Lake 5095, SA, Australia.

E-mail: ysugi@gifu-u.ac.jp (Y.Sugi); ajayan.vinu@unisa.edu.au (A.Vinu)

Figure S1. The Effects of reaction temperature on the conversion of BP in the isopropylation of BP over alkaline earth metal modified MORs.

Figure S2. The Effects of reaction temperature on the selectivities for 4,4'-DIPB in the isopropylation of BP over alkaline earth metal modified MORs. 


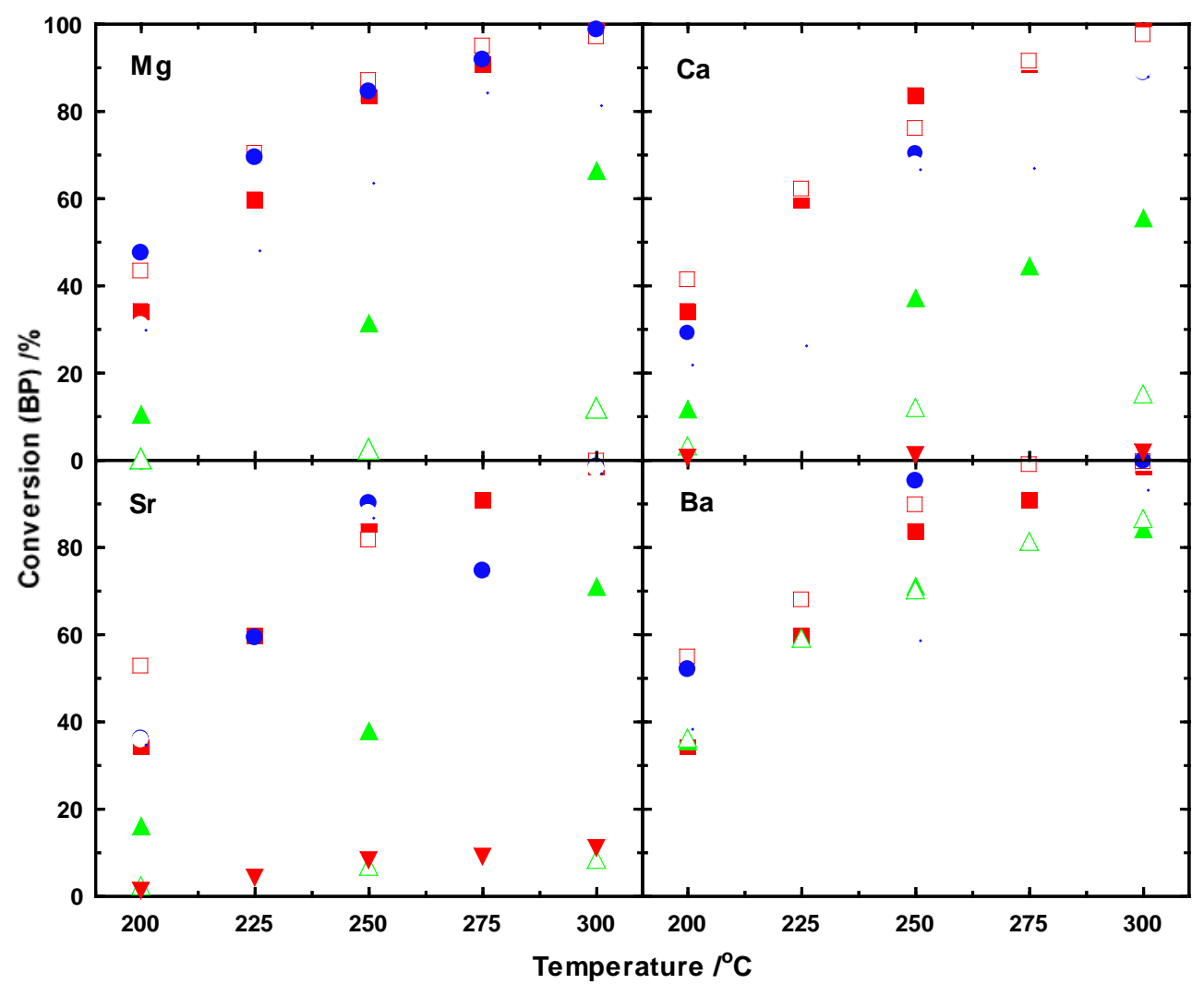

Figure S1. The Effects of reaction temperature on the conversion of BP in the isopropylation of BP over alkaline earth metal modified MORs. Reaction conditions: BP/MOR: 200; temperature: 200-300 ${ }^{\circ} \mathrm{C}$; pressure: $0.8 \mathrm{MPa}$; Period: $4 \mathrm{~h}$. Legends: п:MOR; MgMOR: $\square: 0.1 ; \bullet: 0.2 ; 0: 0.4 ; \triangle: 0.8 ; \triangle:$ 2.0. CaMOR: $\square: 0.05 ; \bullet: 0.125 ; 0: 0.2 ;$ $\triangle: 0.5 ; \triangle: 1.25 ; \nabla: 2.0$. SrMOR: $\square: 0.05 ; \bullet: 0.1 ; 0: 0.2 ; \triangle: 0.6 ; \triangle: 1.0 ; \nabla: 2.0$. BaMOR: $\square:$ $0.07 ; \bullet: 0.15 ; 0: 0.36 ; \Delta: 1.0 ; \triangle: 2.0$. Loadings are based on $1 \mathrm{~g}$ of unmodified MOR. 


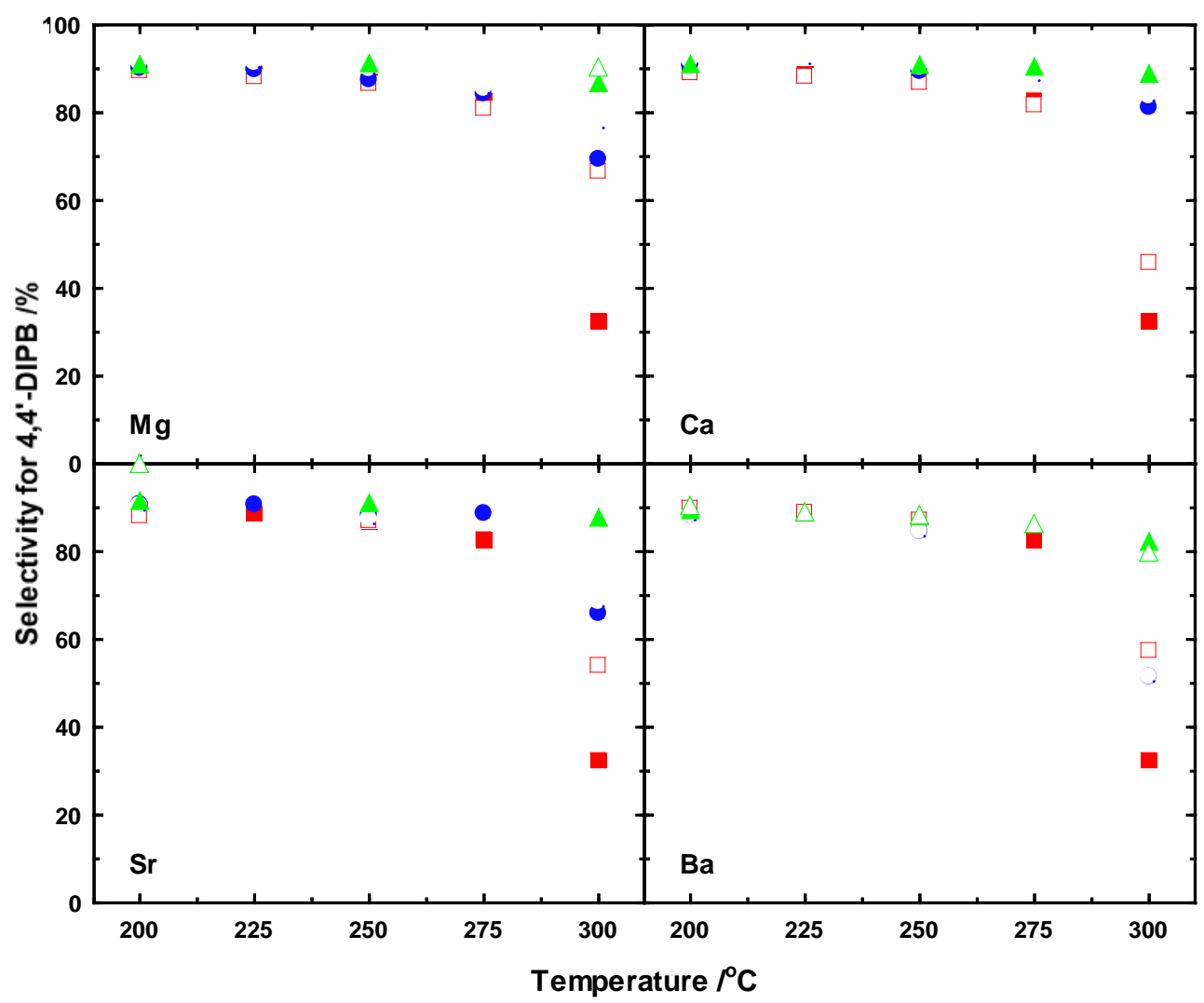

Figure S2. The Effects of reaction temperature on the selectivities for 4,4'-DIPB in the isopropylation of BP over alkaline earth metal modified MORs. Reaction conditions: BP/MOR: 200; temperature: $200-300{ }^{\circ} \mathrm{C}$; pressure: $0.8 \mathrm{MPa}$; Period: $4 \mathrm{~h}$. Legends: see Figure S1. 